\section{Fortbildungs- veranstaltungen}

\author{
Hamburger Augenärztliche \\ Fortbildung: \\ 30. November 2013: \\ Kinderophthalmologie \\ Universitätklinik Hamburg-Eppendorf \\ Info: augenklinik@uke.uni- \\ hamburg.de
}

Münsteraner Fortbildung für

Augenärzte:

11. September 2013

4. Dezember 2013

Klinik und Poliklinik für Augenheilkunde Info: stephan.grewe@ukmuenster.de

\section{Kongresskalender}

\section{8.-31. August 2013 - LOCARNO} 106. Jahreskongress der Schweizerischen Ophthalmologischen Gesellschaft (SOG) Info: www.sog-sso.ch

\section{4.-7. September 2013 - MARSEILLE European Strabismological Association (ESA) \\ Info: www.esa-2013.org \\ 19.-21. September 2013 - BARCELONA Annual Meeting of the Euro- pean Society of Ophthalmic Plastic and Reconstructive Surgery \\ Info: www.esoprs.eu}

19.-22. September 2013 - BERLIN 111. Kongress der Deutschen Ophthalmologischen Gesellschaft DOG

Info: geschaeftsstelle@dog.org
26.-29. September 2013 - HAMBURG $13^{\text {th }}$ EURETINA Congress Info: euretina@euretina.org

\section{September 2013 - HAMBURG} Jahrestagung der Retinologischen Gesellschaft Info: www.retinologie.org

\section{5.-9. Oktober 2013 - AMSTERDAM} XXXI Congress of the European Society of Cataract and Refractive Surgeons ESCRS Info: escrs@escrs.org

\section{7.-11. Oktober 2013 - MÜNCHEN} Refraktionskurs (Schoberkurs) Berufsverband der Augenärzte Deutschlands BVA

Info: thull@augeninfo.de

16.-19. November 2013 - NEW

\section{ORLEANS (LA)}

American Academy of Ophthalmology (AAO) Annual Meeting

Info: meetings@aao.org

27.-30. November 2013 - ROM $93^{\text {th }}$ National Congress of the Italian Ophthalmological Society

Info: congressi@sedesoi.com

\section{November 2013 - ESSEN}

44. Wacker-Kurs für klinische Retinologie

Zentrum für Augenheilkunde, Universitätsklinikum Essen, Prof. Dr. Norbert Bornfeld Info: norbert.bornfeld@uniduisburg-essen.de

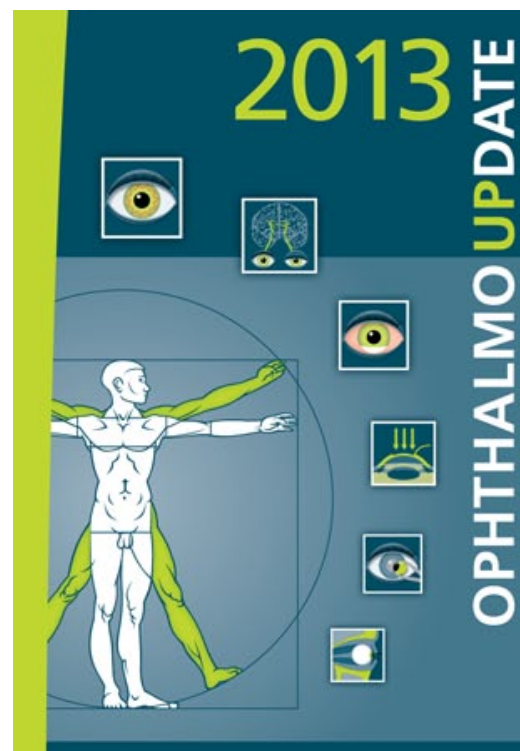

3. Ophthalmologie-Update-Seminar 22. und 23. November 2013, Mainz

- Topaktuelle Studien

- Abdeckung des gesamten Fachbereichs

- Studienüberblick kompakt in zwei Tagen

- Praxisrelevante Studienergebnisse

- Interaktive Diskussionen

- cME-Zertifizierung

- Handbuch mit allen Studienergebnissen

- Media-Präsentation aller Vorträge

Wissenschaftliche Leitung Thomas Reinhard, Freiburg Norbert Bornfeled, Essen
Carsten Framme, Hannover

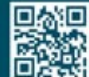

Anmeldung und weitere Informationen unter www.ophthalmo-update.com

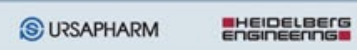

Q Springer Medizin wkonect med 